\title{
Bildung und Altern
}

\section{$Z f E$}

\author{
Klaus Schömann
}

\section{Sammelrezension zu:}

1. Vern L. Bengtson/Daphna Gans/Norella M. Putney/Merril Silverstein (Eds.): Handbook of Theories of Aging. $2^{\text {nd }}$ ed. New York: Springer Publ. 2009. 790 S. ISBN 9780-8261-6251-9 Preis: ca. \$69,-.

2. Fergus I. M. Craik/Timothy A. Salthouse (Eds.): The Handbook of Aging and Cognition. $3^{\text {rd }}$ ed. New York: Psychology Press 2008. 657 S. ISBN 978-0-8058-5990-4. Preis: ca. \$118,-.

3. Sylvia Kade: Altern und Bildung. Eine Einführung. 2., akt. und überarb. Aufl. Bielefeld: W. Bertelsmann 2009. 243 S. ISBN 978-3-7639-3336-5. Preis: 24,90€.

4. Rudolf Tippelt/Bernhard Schmidt/Simone Schnurr/Simone Sinner/Catharina Theisen (Hrsg.): Bildung Älterer. Chancen im demografischen Wandel. Bielefeld: W. Bertelsmann 2009. 223 S. ISBN 978-3-7639-1971-0. Preis: 44,90€.

Das Altern genauso wie die Bildung sind große Themengebiete, die meistens für sich alleine untersucht werden. Zumindest lassen sich Publikationen in der großen Mehrheit der einen oder der anderen Wissenschaftsdisziplin zuordnen. In diesem Fall wären das die Bildungs- bzw. die Altersforschung. Selten gelingt eine Verbindung beider Forschungsausrichtungen, da die Forschungsfelder sich rasch in weitere Subdisziplinen, wie etwa die ökonomische, soziologische, psychologische Bildungs- und Altersforschung, verästeln.

Diese Sammelrezension hat sich das Ziel gesetzt, möglichst umfassende Bearbeitungen, die den jeweiligen Disziplinen gerecht werden, zu besprechen. Die Auswahl fiel auf Überblicksbände, da dort eine multidisziplinäre Herangehensweise am ehesten angetroffen wird. Als roter Faden durch die Rezensionsexemplare bietet sich der Weg von der Theorie oder Theoriekonstruktion, über die zu beobachtenden und zu vermeidenden kognitiven Veränderungen, hin zu dem Einfluss der Institutionen und des gesellschaftlichen Kontextes an. Die umfassende Studie zur Bildung Älterer in Deutschland zeigt

Online publiziert: 19.12 .2009

(C) Die Autoren 2009. Dieser Artikel ist auf Springerlink.com mit Open Access verfügbar.

Prof. Dr. K. Schömann $(\bowtie)$

Jacobs Center on Lifelong Learning and Institutional Development, Sociology Unit

Jacobs University Bremen, College Ring 2, 28759 Bremen, Deutschland

P.O. Box 70561, 28725 Bremen, Tel.: +49-421-200-4750

E-Mail: k.schoemann@jacobs-university.de 
abschließend, wie eine besonders gelungene Verknüpfung beider Themen in der empirischen Bearbeitung aussehen kann.

Bengtson et al. (Eds.), Handbook of Theories of Aging. Diese Publikation hat den Anspruch, einen breiten Überblick über die Theorien, die Theoriebildung und einflussreiche Modelle der Alterung zu dokumentieren. Auch wenn der eindrucksvolle Band nicht vordergründig von Bildung und Altern handelt, so werden gerade die grundlegenden Theorien und Begriffe beider Forschungsfelder ausführlich dargestellt. Im Vorwort werden die acht Teile kurz vorgestellt: Kontext der Theorien, Theoriebildung disziplinenübergreifend, biologische Theorien, psychologische Theorien, sozialwissenschaftliche Perspektiven, Gesellschaft und Politik, Implementation der Theorien in die Praxis und die Zukunft der Theoriebildung.

Das einleitende erste Kapitel der Editoren betont die Bedeutung von Theorien, um Erklärungen zu generieren sowie den Prozesscharakter der Theoriebildung selbst. Der Überblick über die acht Theoriefelder befasst sich bereits mit den grundlegenden Fragestellungen, wie z.B. dem Konzept der Zeit und der Veränderung. Theorien des Alterns werden seit Aristoteles und Cicero eingeordnet nach der Art der Fragestellung in Theorien, die die Variabilität im Altersprozess erklären (Why? How?), und in Theorien, die sich auf einzelne Populationen richten (Who?). Die erste Frage wird in Kapiteln über Evolution und Biodemografie bearbeitet. Die zweite Frage nach dem „Wie“ hat bereits stärkere Verbindung zu Bildungsprozessen und befasst sich daher stärker mit biologischen und psychologischen Herangehensweisen des Alterns. Die Frage nach dem „Wer“ altert schneller, wird von psychologischen und weiteren sozialwissenschaftlichen Ansätzen beleuchtet. Die jeweiligen Schnittstellen von Bildung und Altern werden jedoch nur deutlich, wenn die Fachbegriffe der einzelnen Disziplinen bereits geläufig sind. So ist es für die Erschließung der Schnittmenge der Forschungsfelder wichtig zu wissen, dass Bildung und Alter beispielsweise in der psychologischen Forschung unter den Begriffen der kognitiven Plastizität (Kapitel 17) sowie der Entwicklung über die Lebensspanne bearbeitet werden. Die Soziologen dagegen verwenden den Begriff der Lebensverlaufsforschung und meinen damit die Bedeutung, zwischen individuellem Lebensverlauf, Kohortenprägung und zeitgeschichtlichen Periodeneffekten zu unterscheiden (Kapitel 21).

Eine weitere Theorie mit Bedeutung für Bildung und Altern ist die „dynamic integration theory“ (Kapitel 16). Sie vereint die Perspektive der Kognition mit der Perspektive der Entwicklung der Regelung von Emotionen. Besonders für Ältere ergibt sich daraus ein dynamisches Verhältnis, das Lernprozesse auf vielfältige Art im Alter beeinflusst. Die kumulative Ungleichheitstheorie (Kapitel 22) versteht Ungleichheit als sozialen Prozess, der zu ungleichen Ergebnissen führt und sich über den Lebensverlauf verstärkt. Die kumulative Bildungsselektivität der Weiterbildung entspricht weitestgehend dieser Theorie. Es ist wohl deutlich geworden, dass sich dieses Handbuch eher an die Experten des Feldes richtet, aber von solchen sicher mit Gewinn gelesen wird.

Craik/Salthouse (Eds.), The Handbook of Aging and Cognition. Die dritte Auflage des sehr erfolgreichen Handbuchs ist ein komplett neuer Band. Einige Themen wie Aufmerksamkeit, Gedächtnis und Sprachentwicklung waren und sind auch wieder vertreten, aber besonderes Kennzeichen dieses Bandes ist die Aufnahme von Ergebnissen der 
Neurowissenschaften in systematischer Art und Weise. Das erste Kapitel von Dennis und Cabeza bietet einen hervorragenden Überblick zu den Befunden mit bildgebenden Verfahren des Gehirns und setzt sie in Beziehung zu den wichtigsten Theorien des kognitiven Alterns. Es gilt demnach als gesichert, dass einem Verringern der neuronalen Aktivitäten in occipitalen und mediotemporalen Arealen des Gehirns mit dem Alter, eine Zunahme in frontalen Arealen gegenübersteht. Bei älteren Personen können Verluste in neuronalen Aktivitäten in einer Gehirnregion durch die Verwendung von zusätzlichen Ressourcen in anderen Gehirnregionen kompensiert werden (S. 25). Offen bleibt noch, wie und wann ältere Erwachsene diese Reservekapazitäten einsetzen. Die dominierenden Defizittheorien der Kognitionsforschung (sensorische, Ressourcen, Geschwindigkeit, Inhibition, Erinnerung) werden dann mit den neuesten Ergebnissen der Neurowissenschaften in Einklang gebracht.

Die weiteren Kapitel handeln von genetischen Einflüssen auf die Kognition im Alter, der Neuropsychologie in der Entwicklung von Alzheimer, der Reservekapazität innerhalb des Gehirns, welche eine Schutzfunktion gegenüber den negativen Einflüssen von Altern und Krankheit ausfüllt. Konventionelle Themen wie Aufmerksamkeit, Arbeitsgedächtnis, Sprachentwicklung, Wissen, intra-individuelle Variabilität und Entwicklung über die Lebensspanne machen diese dritte Ausgabe des Handbuchs zu einem wichtigen Referenzband als Grundlage für Forschung zu Altern, Kognition und Bildung. Insgesamt zeichnet der Band ein positiveres Bild des Alterns und der Bildungsprozesse, da die Autoren in mehreren Kapiteln berichten, dass beispielsweise Geschwindigkeitsverluste deutlich sind, aber fast kein Verlust bei der Akkuratheit von Antworten zu verzeichnen ist (S. 507). Tests zum Wortschatz zeigen ebenso die Erweiterungsfähigkeit bis ins mittlere Alter und erst ein sehr spätes Nachlassen (S. 567).

Das Handbuch richtet sich an Experten, die einen detaillierten Überblick über die neuesten Entwicklungen zur Kognition inklusive der Ergebnisse der Neurowissenschaften suchen. Das ist wirklich gelungen. Allerdings: Selbst die Aufnahme von farbigen Darstellungen aus der funktionalen Magnetresonanztomografie (fMRT) erleichtert Einsteigern in die Materie die Lektüre nur wenig.

Kade, Altern und Bildung. Wie der Untertitel dieses Buches bereits klarstellt, handelt es sich bei der zweiten, aktualisierten und überarbeiteten Auflage des Buches um eine auf die deutschsprachige Leserschaft zielende Einführung in das Thema Altern und Bildung. Der Gegenstand wird in seiner ganzen Breite bearbeitet und es wird sorgfältig auf leicht verständliche Begriffsdefinitionen geachtet. Kritische Einschätzungen aus persönlicher Sicht, die Leser eventuell nicht immer teilen werden, werden kombiniert mit einer Fülle von hilfreichen Verweisen auf deutschsprachige Hintergrundliteratur aus Wissenschaft, Ministerien und Trägern der Altersbildung in Deutschland. Mit lediglich vier englischsprachigen Verweisen bei über 400 Einträgen im Literaturverzeichnis bietet das Buch einen deutlichen Kontrast zu den oben besprochenen Handbüchern. Dies unterstreicht den Einführungscharakter in das Thema sowie die Praxistauglichkeit für Akteure der Altersbildung. Abstriche in Bezug auf die Verwendung der aktuellsten wissenschaftlichen Befunde, beispielsweise aus dem Bereich der Kognition, aber auch aus dem Bereich der Demografie, werden in Kauf genommen. So ist die Tabelle (S. 25) zu den Erwerbsquoten nach Alter und Geschlecht mit Werten von 1975, 1985, 1998 arg veraltet und doch im 
Zeitalter des Internets so leicht zu aktualisieren (Eurostat 2009). Es ist der Politik und Wirtschaft allerdings, anders als von der Autorin behauptet (S. 24: „Doch ist der Rückgang der Erwerbsquote ... Resultat ... einer gezielten Politik der Frühverrentung seit den 80er Jahren“), zumindest ein Anfang gelungen, den Trend der Frühverrentung umzukehren (Eurostat 2007).

Die Stärken dieser Einführung liegen sicherlich in der breiten und kritischen Anlage. Themen wie die Pluralisierung der Lebensstile im Alter werden knapp und verständlich an Beispielen „Altern auf dem Lande“ und „Altern in der Fremde“ verdeutlicht. Die Kapitel zur Institutionalisierung der Altersbildung, der differenziellen Bildung durch plurale Lernmodelle, dem Strukturwandel des Lernens und zur Bildungsdidaktik sind mit den kursiv gesetzten Merksätzen für Einsteiger ansprechend aufbereitet.

Tippelt et al. (Hrsg.), Bildung Älterer. Die neueste repräsentative wissenschaftliche Basis zur Bildung Älterer in Deutschland bietet die vom Bundesministerium für Bildung und Forschung geförderte Studie „EdAge“ von Tippelt und anderen, die in der Reihe „DIE spezial“ des Deutschen Instituts für Erwachsenenbildung erschienen ist. In vorbildlicher Weise werden dort Ergebnisse einer neuen repräsentativen Befragung mit aufwendig durchgeführten Ergebnissen aus verschiedenen qualitativen Ansätzen dokumentiert. Das erste Kapitel, Ausgangspunkt und Anlage der Studie, beschreibt kurz die demografische Konstellation und ausführlich den aufwendigen methodischen Aufbau der Studie. Die folgenden Kapitel berichten dann aus den Ergebnissen der beiden Teile. In der repräsentativen Studie, beispielsweise, wurden in 2007 fast 5.000 Personen interviewt, die zwischen 45 und 80 Jahre alt waren.

Ausführlich wird das derzeitige Bildungsverhalten Älterer in Kapitel 2 dargestellt, einschließlich der Erwartungen von und Barrieren für Ältere sowie dem für Ältere so wichtigen Bereich des informellen Lernens. Ausführliche Befunde zu Lernfeldern und vielfache Hinweise für die didaktische Gestaltung von Bildungsangeboten und das abschließende Kapitel zur Zielgruppendifferenzierung machen die Studie zu einem Fundus an neuen Erkenntnissen und praxisrelevanten Hinweisen. Didaktische Hinweise zur Wissensvermittlung fallen dabei sehr detailliert aus. Die Zielgruppendifferenzierung wird demonstriert an der notwendigen Spezifizierung für Männer kurz nach dem Übertritt in die Rente, für VHS-Teilnehmer(innen) mit niedrigem Bildungsabschluss sowie für Personen mit Migrationshintergrund unterschiedlicher Nationalitäten.

Viele Befunde sind neu, weil die Gruppe der 65- bis 80-Jährigen selten vorher systematisch befragt wurde. Das Annähern der Weiterbildungsteilnahmen nach Alter und Erwerbstätigkeit in der ältesten Altersgruppe resultiert einfach aus der geringen Zahl der Erwerbstätigen in dieser Altersgruppe. Informell Lernende unter den lernaktiven Nichterwerbstätigen bleiben allerdings mit ca. 30 Prozent fast gleich hoch unter den Älteren wie in den jüngeren Altersgruppen ab 35 Jahren. Bei den Prädiktoren für die Teilnahme an Weiterbildungsmaßnahmen aus beruflichen sowie privaten Gründen in den letzten 12 Monaten (S. $40 \mathrm{ff}$.) zeigt sich in nicht nur für die Autoren überraschender Weise, dass das Altersbild der Befragten keinen signifikanten Einfluss auf die Teilnahme hat. Die aufwendige Erhebung von außerberuflichen Aktivitäten erweist sich jedoch in den Modellschätzungen als bedeutsamer Prädiktor. 
Interessant ist der Versuch einer neuen Typologie zum Bildungsverständnis und der Bildungsmotivation von Älteren. Die Autoren verweisen dabei selbst auf die zu vermutende Dynamik dieser Typenbildung. Personen werden, in Abhängigkeit von der Lebensphase, zwischen diesen Typen wechseln. Das unterstreicht nun die Bedeutung von Längsschnitterhebungen und deren Analysen (Schömann u. Baron 2009), die trotz dieser eindrucksvollen Erhebung unverzichtbar bleiben.

Dem abschließenden Fazit der Autoren, „Ältere nicht als Objekt von Bildungsmaßnahmen, sondern als gestaltende Subjekte individueller und gesellschaftlicher Entwicklung zu begreifen“ (S. 206), kann nur zugestimmt werden. Die dafür notwendige Informationsbasis ist, nicht nur in dem letzten, sondern in allen hier diskutierten Büchern, ausführlich dargestellt worden.

Open Access Dieser Artikel unterliegt den Bedingungen der Creative Commons Attribution Noncommercial License. Dadurch sind die nichtkommerzielle Nutzung, Verteilung und Reproduktion erlaubt, sofern der/die Originalautor/en und die Quelle angegeben sind.

\section{Literatur}

Eurostat (2007). Der Übergang vom Erwerbsleben in den Ruhestand. In Statistik kurz gefasst. Bevölkerung und soziale Bedingungen Nr. 97/2007. http://www.eds-destatis.de/de/downloads/ sif/sf_07_097.pdf. Zugriff am 24.06.2009.

Eurostat (2009). Strukturindikatoren. http://epp.eurostat.ec.europa.eu/portal/page/portal/structural_indicators/indicators/employment. Zugriff am 24.06.2009.

Schömann, K., \& Baron, S. (2009). Zustandsbeschreibung der Erwachsenenbildung in Deutschland im internationalen Vergleich. In U. M. Staudinger \& H. Heidemeier (Hrsg.), Altern, Bildung und lebenslanges Lernen (Altern in Deutschland, Bd. 2 = Nova Acta Leopoldina NF, Bd. 100, S. 31-41). Halle (Saale): Dt. Akademie der Naturforscher Leopoldina e.V. 